\title{
Severidade clínica e funcionalidade de pacientes hemiplégicos pós-AVC agudo atendidos nos serviços públicos de fisioterapia de Natal (RN)
}

\author{
Clinical severity and functionality of acute stroke patients \\ attended at the physiotherapy public services \\ of Natal, Rio Grande do Norte State, Brazil
}

Fabrícia Azevêdo da Costa ${ }^{1}$

Diana Lídice Araújo da Silva²

Vera Maria da Rocha ${ }^{3}$

${ }^{1}$ Departamento de Fisioterapia, Centro de Ciências da Saúde UniversidadeFederal do Rio Grande do Norte. Av. Salgado Filho 3.000, N ova Descoberta. 59000-000 Natal RN. facnat@yahoo.com ${ }^{2}$ Prefeitura M unicipal de $N$ atal, Secretaria M unicipal deSaúde, Distrito Sanitário Leste.

${ }^{3}$ Departamento deEducação

Física, Escola Superior de Educação Física,

UniversidadeFederal do Rio Grande do Sul.
Abstract Thisarticleinvestigates the severity and functional independence of hemiplegics patients' post-acute stroke by means of a multidisciplinary clinical staff. It is a descriptive study composed by 40 hemi plegics attended at the four largest physiotherapy public services in Natal, Rio Grande do N orte State. The methods used were an evaluation form, Functional Independence $M$ easure and NIHSS. Theresultsshowed a samplepredominantly female (55\%), stroke ischemic ( $90 \%)$, right brain hemisphere $(52.5 \%)$ and $\mathrm{H}$ ypertension Risk Factor $(90 \%)$. The mean of clinical severity and functional independence was $13.32 \pm 4.7$ and $54.6 \pm 17.15$ respectively. There was no significant difference between themean of functionality about stroke side (pvalue $=0.66$ ). There is a significant relation between clinical severity and functional independence $(r=-0.45$ pvalue $=0.003)$. It can be concluded that the level of clinical severity and functional dependence of stroke patients that make use of public physiotherapy services is significant and show the necessity, beyond the classic treatment, to be encouraged preventive educational actions to improve knowledge of this population. Key words Stroke, Functionality, Clinical severity
Resumo 0 acidente vascular cerebral (AVC) re presenta a terceira causa de morte em vários países do mundo e principal causa de incapacidade física. 0 objetivo deste estudo foi investigar por meio de uma equipe multidisciplinar a severidade clínica ea independência funcional de pacientes hemi plégicos pós-AVC. Trata-se de estudo descritivo composto por quarenta hemi plégicos atendidos nos quatro maiores serviços públicos de fisioterapia da cidade de $N$ atal (RN) . Foram utilizadosuma ficha deavaliação, a medida deindependência funcional e o NIHSS. A amostra é predominantemente feminina( $55 \%$ ), AVC isquêmico( $90 \%)$, hemisfério cerebral direito(52,5\%) e fator de risco hipertensão( $90 \%$ ). A média da severidade clínica é $13,32 \pm 4,7$ e da independência funcional, 54,6 17,15. Não houve diferença significativa entre as médias funcionais dos pacientes quanto ao lado do AVC ( pvalor $=0,66$ ). Existiu relação significativa entre severidade clínica e independência funcional $(r=-0,45$ pvalor $=0,003)$. A severidade clínica ea dependência funcional de pacientes com AVC, ao darem entrada na fisioterapia, são significativas e evidenciam a necessidade de, além do tratamento clássico, serem incentivadas condutas educacionais que visem à conscientização da população.

Palavras-chave AVC, Funcionalidade, Severidade clínica 
Introdução

0 acidente vascular cerebral (AVC) éuma alteração da circulação cerebral que ocasiona um déficit transitório ou definitivo no funcionamento de uma ou mais partes do cérebro, podendo ser por meio isquêmico ou hemorrágico e resultando em perda da função neurológica'. O AVC representa a tercei ra causa de morte em vários países do mundo ea principal causa deincapacidadefísica mental, acometendo, particularmente, pessoas com mais de 55 anos $^{2,3}$. No Brasil, segundo dados do Ministério da Saúde, as doenças cardiovasculares são responsáveis por $32 \%$ dos óbitos. Ao se ajustar os dados de mortalidade com a idade, o acidente vascular encefálico (AVE) aparece como líder de causas de morte no Brasil $^{4}$. Essa patologia cerebrovascular atinge com maior incidência pessoas em idade avançada, período da vida em que se observam as maiores taxas de óbito e sequelas. O AVE ocorre em todas as idade, mas a incidência dobra a cada década após os 65 aos de idade ${ }^{2,3}$. Grande parte dos pacientes com AVE evolui com incapacidades e prejuízos sensórios-motores, tendo como consequência um impacto significante em seu nível deindependênciafuncional ${ }^{5}$.

N os serviços de reabilitação, observa-se que a população de pacientes com AVE se concentra predominantemente na faixa etária adulto-ido$\mathrm{SO}^{6}$, período no qual pode ocorrer, naturalmente, a diminuição da acurácia das condições funcionais pelo processo deenvelhecimento. A avaliação da independência funcional destes pacientes deve contemplar a análise das atividades de vida diária que geralmentese encontram afetadas após o AVE ${ }^{5}$, adequando assim a interven ção terapêutica às reais condições do paciente. Esta avaliação é usada para adequar a intervenção terapêutica às reais condições do paciente, comparar os resultados em diferentes momentos da intervenção, melhorar os procedimentos e determinar as necessidades pessoais de cada um.

Esteestudo teve como objetivo investigar por meio de uma equipe multidisciplinar (fisioterapeutas, terapeutas ocupacionais e fonoaudiólogos) a severidade clínica ea independência funcional de pacientes pós-AVC agudo que chegam aos serviços de fisioterapia do SUS da cidade de $\mathrm{N}$ atal (RN). Objetivou-se também verificar a existência de associação entreas variáveis clínicas dos indivíduos investigados e seus desempenhos funcionais.

\section{Métodos}

A pesquisa caracterizou-se por ser um estudo descritivo, visando avaliar a capacidade funcional de indivíduos hemiplégicos pós-AVC. A população foi constituída por pacientes com AVC agudo (atétrês meses de lesão) que deram entrada nos cinco maiores serviços de fisioterapia do SUS da cidade de $N$ atal (setor de fisioterapia do Hospital Universitário O nofre Lopes/UFRN, setor de fisioterapia da Universidade Potiguar do Rio Grande do Norte/UNP, Centro Clínico Asa Norte, Centro Clínico José Carlos Passos e Centro de Reabilitação do Adulto-CRA) no período de maio de 2007 a maio de 2008.

A amostra por contingência foi composta por quarenta indivíduos, admitidos segundo os seguintes critérios de inclusão: (1) possuir diagnóstico de AVC confirmado por exame complementar (TC ou RM ); (2) ter sido atendido pelo serviço de fisioterapia de uma das instituições da pesquisa entre maio/2007 e maio/2008; (3) ter idade entre quarenta e noventa anos; (4) tempo deAVC inferior a três meses e (5) o AVC ser unilateral e não recorrente.

Como critério de exclusão, foi admitido a presença de patologias associadas que pudessem acarretar sequelas funcionais além das provocadas pelo AVC, tais como amputação e doenças crônicas severas (Parkinson, Alzheimer, etc.). Pacientes com afasia grave e distúrbios visuais também foram evitados.

\section{Instrumento de coleta de dados}

Para coleta dos dados gerais dos pacientes, foi utilizada a ficha deavaliação fisioterapêutica, constituída por identificação, condições clínicas, história da doença atual (HDA), antecedentes patológicos efamiliares, hábitos de vida, medicamentos e exame físico (pal pação e inspeção), conforme modelo utilizado pelo Serviço de Fisioterapia do Hospital Universitário Onofre Lopes. Visando avaliar quantitativamente o estado neurológico e o grau de recuperação dos pacientes, foi utilizado o questionário NIH SS - The N ational Institutes of $\mathrm{H}$ ealth StrokeScale. Sua confiabilidadee validade são bem documentadas na literatura ${ }_{7,8}$. Essa escala écomposta por onze itens, que incluem nível de consciência, movimentos oculares, campo visual, movimentos faciais, função motora e ataxia de membros superiores e inferiores, assim como sensibilidade, linguagem, presença de disartria e de negligência espacial. Para uma meIhor avaliação das funções de linguagem e do ní- 
vel de atenção dos pacientes, são utilizadas palavras, sentenças e figuras ${ }^{7,9}$. Com o objetivo de avaliar a independência funcional, foi empregado a medida de independência funcional (MIF).

A MIF é um instrumento composto por dezoito itens queavaliam seis diferentes áreas, compreendendo itens motores e cognitivos, eum sistema de graduação da resposta do paciente que pode variar de 01 a 07 pontos $^{10}$.

Neste trabalho, foi utilizado a medida de independência funcional exclusivamente com itens motores, dispensando assim a parte cognitiva deste protocolo. Portanto, foram analisados apenas as quatro áreas motoras e os seus respectivos itens: cuidados pessoais: 1- comer, 2 -cuidados gerais, 3 - banho, 4 - vestir (externo), 5 - vestir (peças íntimas) e 6 - uso do banheiro; controle dos esfíncteres: 7 - controle vesical e 8 - controle intestinal; mobilidade: 9 -transferência cama/cadeira, 10 transferência no banheiro, 11- transferência no banho e, a última área, da locomoção: 12 - deambulação ou cadeira de rodas e 13 - escada.

A pontuação de 07 em determinado item indica que o paciente é totalmente independente para a atividade, sem necessidade de auxílio ou medicação. Já a pontuação 01 indica total dependência do paciente para desempenhar grande parte da tarefa solicitada. A pontuação mínima que o paciente pode obter na M IF motora é 13 e a sua máxima, 91 pontos ${ }^{11}$.

Procedimentos para coleta de dados

Inicialmente, o projeto de pesquisa foi encaminhado para apreciação do Comitêde Ética em Pesquisa em Humanos - UFRN (CEP-UFRN). Após aprovação de acordo com a Resolução n ${ }^{\circ}$ 196/96, foi realizado um treinamento dos pesquisadores para a utilização dos instrumentos de coleta, a fim de uniformizar os procedimentos. Antes da aplicação do protocolo, cada participanterecebeu informações sobreo estudo, seus objetivos elimitações eassinou um termo de consentimento livre e esclarecido, confirmando sua participação voluntária. Os instrumentos foram aplicados uma única vez em cada participante no início do seu tratamento fisioterapêutico.

Os dados obtidos foram tabulados e submetidos à análise estatística pelo SPSS. Admitiu-se uma distribuição normal dos dados conforme a análise gráfica pelo Normal Probability Plot e um alfa de $5 \%$ de significância para o estudo.

Com o intuito de caracterizar a amostra investigada, aplicou-se a estatística descritiva ( frequências, medias e desvio padrão) e, visando verificar a existência dediferença significativa entreas medias funcionais quanto aos lados do AVC e a existência derelação entrea severidadeclínica eo estado funcional dos pacientes, aplicou-se a estatística inferencial (testet-Student para amostrasindependentes e correlação de Pearson, respectivamente).

\section{Resultados}

A amostra investigada foi caracterizada de acordo com os aspectos sociodemográficos (gênero, estado civil, idade e nível de escolaridade) e os aspectos clínicos (etiologia do AVC, hemisfério cerebral acometido, dominância motora manual, tempo de AVC, tempo de tratamento fisioterapêutico, tempo entrea lesão eo início do tratamento e medicamentos). Os resultados encontrados apontaram para uma amostra de quarenta pacientes predominantemente feminina (55\%), sendo o estado civil mais prevalente 0 casado (50\%). A média de idade dos indivíduos foi de 65,9 anos $( \pm 10,9)$. A proximadamente $68 \%$ da amostra encontravam-se na faixa etária entre quarenta e setenta anos. 0 nível de escolaridade predominante foi o ensino fundamental incompleto $(n=15,37,5 \%)$ (Tabela 1 ).

Tabela 1. Caracterização do grupo de pacientes pós-AVC agudo quanto aos aspectos sociodemográficos. Natal (RN) 2008.

\begin{tabular}{|c|c|}
\hline Variáveis & Pacientes $(n=40)$ \\
\hline \multicolumn{2}{|l|}{ Gênero } \\
\hline & $\begin{array}{l}\text { Feminino }=22(55 \%) \\
\text { Masculino }=18(45 \%)\end{array}$ \\
\hline \multicolumn{2}{|l|}{ Estado civil } \\
\hline & Divorciado = $02(05 \%)$ \\
\hline & Solteiro $=04(10 \%)$ \\
\hline & Viúvo= $13(32,5 \%)$ \\
\hline & Casado $=20(50 \%)$ \\
\hline \multicolumn{2}{|l|}{ Escolaridade } \\
\hline & Analfabeto = $13(32,5 \%)$ \\
\hline & Ensino fundamental incompleto $=15(37,5 \%)$ \\
\hline & Ensino fundamental completo $=04(10 \%)$ \\
\hline & Ensino médio completo $=7(17,5 \%)$ \\
\hline & Ensino superior $=01(2,5 \%)$ \\
\hline \multicolumn{2}{|l|}{ Dominância m. manual } \\
\hline & Direita = $35(87,5 \%)$ \\
\hline & Esquerda $=05(12,5 \%)$ \\
\hline \multicolumn{2}{|l|}{ Idade - avaliação } \\
\hline & $40-60$ anos $=13(32,5 \%)$ \\
\hline & $61-70$ anos $=14(35 \%)$ \\
\hline & $71-80$ anos $=8(20 \%)$ \\
\hline & $81-90$ anos $=(12,5 \%)$ \\
\hline
\end{tabular}


Com relação às características clínicas, a etiologia do AVC mais prevalente foi o AVC isquêmico ( $90 \%)$, sendo o hemisfério cerebral direito mais acometido (52,5\%). Quanto à lateralidade, $87,5 \%$ dos pacientes investigados exibiam dominância motora manual à direita, sendo $52,5 \%$ a porcentagem de pacientes que apresentaram hemiplegia homolateral à dominância motora manual. A hipertensão arterial sistêmica foi 0 fator de risco preponderante, estando presente em $90 \%$ dos pacientes investigados.

Considerando a questão medicamentosa, observamos que $90 \%$ dos pacientes faziam uso de anti-hipertensivo e $41 \%$ medicavam-se com algum tipo de antiagregante plaquetário. A maior parte dos pacientes deste estudo (75\%) foram

Tabela 2. Caracterização do grupo de pacientes pós-AVC agudo quanto aos aspectos clínicos. Natal (RN), 2008.

\begin{tabular}{|c|c|}
\hline Variáveis & Pacientes $(n=40)$ \\
\hline \multicolumn{2}{|l|}{ Etiologia do AVE } \\
\hline & Isquêmico = 36 (90\%) \\
\hline & Hemorrágico $=04(10 \%)$ \\
\hline \multicolumn{2}{|l|}{ Hemisfério cerebral } \\
\hline \multirow[t]{2}{*}{ acometido } & Direito $=21(52,5 \%)$ \\
\hline & Esquerdo= $19(47,5 \%)$ \\
\hline \multicolumn{2}{|l|}{ Fator de risco } \\
\hline & Hipertensão = 36 (90%) \\
\hline & Diabetes mellitus = $14(35 \%)$ \\
\hline & Tabagismo $=10(25 \%)$ \\
\hline & Cardiopatia $=10(25 \%)$ \\
\hline & Etilismo $=09(22,5 \%)$ \\
\hline \multicolumn{2}{|l|}{ M edicamentos } \\
\hline & Anti-hipertensivo = 36 (90\%) \\
\hline & Antiagregante plaquetário $=22(41 \%)$ \\
\hline & Diurético = $05(12,5 \%)$ \\
\hline & Antidiabético = $03(7,5 \%)$ \\
\hline & Antidepressivo $=02(5 \%)$ \\
\hline & Antipsicótico = $02(5 \%)$ \\
\hline & Ansiolítico = $01(2,5 \%)$ \\
\hline \multicolumn{2}{|l|}{ Tempo de sequela } \\
\hline & $01-30$ dias $=13(32,5 \%)$ \\
\hline & $31-60$ dias $17(42,5 \%)$ \\
\hline & $61-90$ dias $=10(25 \%)$ \\
\hline \multicolumn{2}{|c|}{ Tempo lesão - fisioterapia } \\
\hline & $01-30$ dias $=27(67,5 \%)$ \\
\hline & $31-60$ dias $09(22,5 \%)$ \\
\hline & M ais de 60 dias $=04(10 \%)$ \\
\hline \multicolumn{2}{|l|}{ Frequência do TTO } \\
\hline & $1 x /$ semana $=16(40 \%)$ \\
\hline & $2 x /$ semana $=22(55 \%)$ \\
\hline & $3 x /$ semana $=02(05 \%)$ \\
\hline
\end{tabular}

avaliados no intervalo deatédois meses pós-AVC (média 44 dias, $\pm 23,6$ ). A média do tempo entre AVC e o início do tratamento fisioterápico foi $27,12( \pm 22,04)$, com $67,5 \%$ da amostra buscando atendimento fisioterapêutico no período de até um mês pós-AVC. A maior parte dos pacientes $(n=22,55 \%)$ realizavam tratamento fisioterápico duas vezes por semana (Tabela 2 ).

Quanto à severidade clínica do AVC, nesta pesquisa, foi observado o predomínio dos pacientes apresentando nível moderado de comprometimento ( $n=23,57,5 \%$ ), seguido pelo nível grave $(n=17,42,5 \%)$, sendo a severidade média observada $13,32 \pm 4,7$. Já com relação ao nível de independência funcional, foi constatado que a média do escore total referente à M IF motora neste estudo foi de $54,6 \pm 17,15$, valor este compatível com comprometimento funcional moderado; não houve diferença estatisticamente significativa entre as médias funcionais dos pacientes quanto ao lado do AVC (AVC direito 53,5 $\pm 17,35 \times 55,94 \pm 17,31$ p valor $=0,66$ ) (Tabela 3).

Por fim, ao analisarmos a existência de relação entre a severidade clínica do AVC eo nível de independência funcional, foi verificada a presença de uma relação negativa e significativa com valor de $r=-0,45 p$ valor $=0,003$ (Figura 1 ) .

\section{Discussão}

Este estudo teve como objetivo investigar, por meio de uma equipemultidisciplinar, a severidadeclínica ea independência funcional de pacientes pós-AVC agudo que chegam aos serviços de fisioterapia do SUS da cidade de $N$ atal (RN). Diante dos resultados encontrados neste trabal ho, foi observado que a maior parte das pessoas acometidas por AVC eram do sexo feminino e casadas. Alguns estudos verificaram queo gênero dos pacientes não estava associado com o maior grau de incapacidade dos mesmos pós-AVC ${ }^{12}$.

Já com relação à etiologia do AVC, constatouse a maior prevalência do tipo isquêmico, o que está de acordo com os estudos observados na literatura ${ }^{6,13}$. Num estudo desenvolvido em 2003 sobrerecuperação funcional depacientes com AVC isquêmico e hemorrágico, foi encontrado que, embora o AVC hemorrágico seja o tipo menos frequente, correspondeà forma mais grave, o que consequentementeacarreta maiores complicações neurológicas para os pacientes quando comparados com o do tipo isquêmico ${ }^{14,15}$. A pesar disso, este tipo de AVC apresenta melhor prognóstico funcional durante a reabilitação, talvez devido ao 
Tabela 3. Severidadeclínica (NIHSS) eindependência funcional (MIF) quanto ao lado da lesão de pacientes pós-AVC agudo. Nata (RN), 2008.

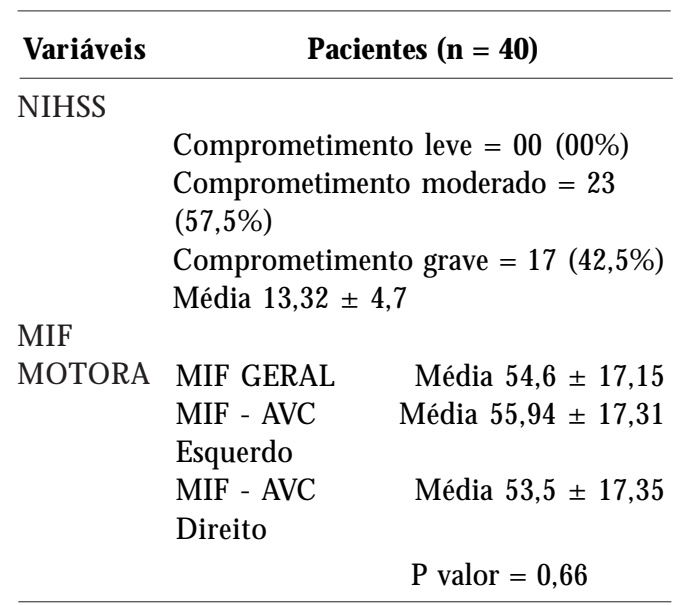

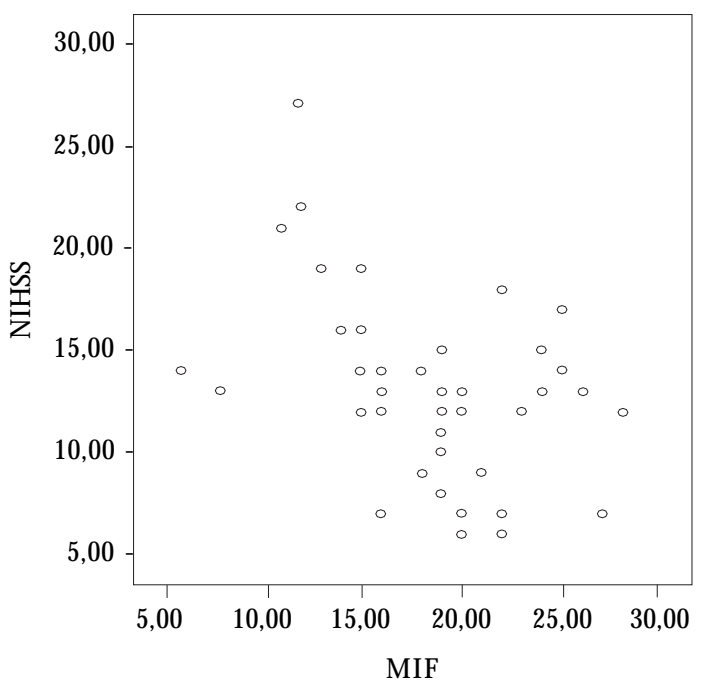

$r=-0,45 ;{ }^{*} p$ valor $=0,003$.

Figura 1. Correlação entre a severidade clínica (NIHSS) e a independência funcional (MIF) de pacientes pós-AVC agudo. Natal (RN), 2008.

seu mecanismo de lesão. Este achado também foi encontrado em outro estudo ${ }^{16}$.

Quanto ao lado cerebral, observou-se nesta pesquisa que o hemisfério direito foi o mais aco- metido; entretanto, vários estudos apontam o hemisfério cerebral esquerdo como mais prevalente, apesar da diferença de prevalência entre os dois hemisférios não ser muito discrepante nestes estudos ${ }^{6,17,18}$. A média de idade na data da avaliação foi de 65,9 anos $( \pm 10,9)$, havendo uma maior concentração de paciente neste estudo no intervalo entre quarenta e setenta anos. Alguns autores apontam que existe uma maior prevalência de pacientes com AVC no intervalo entre 61 e oitenta anos. Entretanto, observa-se que, nos países em desenvolvimento, o AVC ocorre uma década mais cedo do que em países desenvolvidos. Esse fato é atribuído à reduzida expectativa de vida daqueles que vivem em condições sociais desfavorávei ${ }^{13}$. Estudos brasileiros também encontraram esses valores médios de idade dos pacientes com AVC ${ }^{6,19}$. A hipertensão arterial sistêmica (HAS) apresentou-se neste estudo como o principal fator de risco, corroborando com o estudo de Pires et al. ${ }^{20}$, no qual foi verificado que $87,8 \%$ dos pacientes com AVC eram hipertensos e esta alta frequência se justifica, uma vez que, segundo o autor, um dos principais fatores de risco para HAS é idade superior a sessenta anos, muito embora já tenha se encontrado alta frequência de HAS entre pacientes com idades entre quinze 49 anos.

Visando avaliar o nível de severidade clínica em que se encontravam os pacientes ao darem entrada nos serviços de fisioterapia do SUS, foi aplicado o NIHSS. Os resultados encontrados com este enfoque apontaram a inexistência de pacientes com nível de comprometimento leve ao início do tratamento fisioterapêutico, evidenciando as implicações clínicas que esta patologia acarreta na vida desta população.

Segundo Schlegel et al. ${ }^{7}, 0$ escorefinal encontrado no NIH SS na data da admissão do paciente à terapia pode ajudar no planejamento da recuperação do doente, indicando a necessidade de cuidados a longo prazo que esse paciente precisará. Ainda de acordo com este autor, mais de $80 \%$ dos pacientes com pontuação inferior a 5 no momento da admissão receberão alta rapidamente sem maiores intercorrências. Já aqueles com pontuação entre 6 e 13 costumam exigir um programa de reabilitação mais elaborado e, por fim, os demais pacientes, com pontuação de 14 ou superior, frequentemente precisam de cuidados reabilitativos mais intensivos epor um período de tempo mais prolongado.

Nesta pesquisa, foi observado o predomínio dos pacientes que dão entrada no serviço de fisioterapia apresentando nível moderado de seve- 
ridade clínica seguido pelo nível grave. Este fato nos aponta para a necessidade de assistência que estes pacientes vão requerer do SUS, bem como a quantidade de tempo que permanecerão no serviço, impossibilitando assim a entrada de novos pacientes no sistema.

Com relação ao nível deindependência funcional dos pacientes, foi observado, por meio do instrumento MIF, que a média do escore total referente à MIF motora neste estudo foi de $54,6 \pm 17,15$, valor sugestivo também de nível moderado de comprometimento funcional. Estes achados estão de acordo com o estudo de validação da M IF no Brasil, que encontrou valores médios de 54,1 $\pm 23,00$ para pacientes com lesões encefálicas no início do processo de reabilitação ${ }^{10}$. De acordo com este autor, nos pacientes com lesões encefálicas, os valores da M IF motora apresentaram associação com o dimídio afetado, evidenciando nos pacientes com comprometimento predominante em hemicorpo esquerdo uma pior performance nas tarefas motoras. Demais pesquisadores também encontraram esses resultado ${ }^{21}$. Corroborando com estes achados, no presente trabalho foi encontrado queos pacientes com AVC direito (comprometimento do hemicorpo esquerdo) exibiram média funcional inferior a pacientes com AVC esquerdo. Entretanto, esta diferença não foi estatisticamente significante, talvez devido ao menor número amostral do presente trabalho quando comparado ao estudo referido.

Por fim, ao ser investigado se a severidade clínica do AVC apresentava al guma relação com o nível deindependência funcional dos pacientes ao darem entrada no serviço de fisioterapia, foi verificada a presença de uma relação negativa e bastante significativa, apontando que quanto mais grave o nível deseveridadeclínica dos pacientes, pior suaindependência funcional; portan- to, maior a dependência destes indivíduos para realização de atividades da vida diária (AVD).

Este fato é de grande relevância clínica e administrativa para os serviços de saúde,uma vez que verificamos que em todas as instituições de coleta de dados existia lista de espera de pacientes pós-AVC para tratamento e, como os pacientes estão chegando com tal nível de comprometimento, acredita-se que o tempo para alta dos mesmos tende a ser razoavelmente longo, inviabilizando, assim, a assistência aos demais pacientes recém-acometidos por esta patologia.

0 presente trabalho apresenta, entretanto, uma certa limitação, uma vez que não foram analisadas as possíveis influências que a extensão e a topografia da lesão poderiam trazer para o estado funcional dos pacientes investigados. No entanto, como foi aplicado o protocolo NIHSS eciente deque o foco desteinstrumento é a avaliação da severidade do quadro clínico do paciente pós-AVC, acredita-se que esta limitação seja pouco relevante para o estudo. Portanto, esperamos que os resultados encontrados nesta pesquisa possam contribuir para estudos futuros que contemplem a funcionalidade de paciente pós-AVC nos serviços públicos de saúde, visando à elaboração de abordagens terapêuticas condizentes com o quadro destes pacientes enorteando profissionais que trabalhem com esta população específica.

Os achados encontrados sugerem que a severidade do quadro clínico e o comprometimento na função de pacientes com AVC ao darem entrada no SU S são significativos e evidenciam a necessidade de, além do tratamento fisioterapêutico clássico, serem incentivadas intervenções precoces na população em geral por meio de condutas educacionais quevisem uma melhor conscientização e prevenção desta patologia. 


\section{Colaboradores}

FA Costa trabalhou em todas as etapas da elaboração do artigo (concepção, coleta, análise dos dados e redação final do trabalho); DLA Silva, na pesquisa bibliográfica e redação final do trabalho e VM Rocha, na revisão crítica e redação final do trabalho.

\section{Referências}

1. Rafii MS, Hillis AE. Compendium of cerebrovascular diseases. Int Rev Psychiatry 2006; 18(5):395-407.

2. Ingall T. Stroke - incidence, mortality, morbility and risk. J Insur med 2004; 36(2):143-152.

3. Pires SL, Gagliardi RJ, Gorzoni ML. Estudo das frequências dos principais fatores de risco para Acidente Vascular Cerebral em idosos. Arq Neuropsiquiatr 2004; 62(3B):844-851.

4. Lotufo PA. Stroke in Brazil: a neglected disease. Sao Paulo M ed J. 2005; 123(1):3-4.

5. Falcão IV, Carvalho EM F, Barreto KML, Lessa FJD, Leite VMM. Acidente vascular cerebral precoce: implicações para adultos em idade produtiva atendidos pelo Sistema Ú nico de Saúde. Rev. Bras. Saude Mater. Infant. 2004; 4(1):95-102.

6. Minelli C, Fen LF, M inelli DP. Stroke Incidence, Prognosis, 30-Day, and 1-Year Case Fatality Rates in Matão, Brazil - A Population-Based Prospective Study. Stroke 2007; 38(11):2906-2911.

7. Schlegel D, Kolb SJ, Luciano JM, Tovar JM, Cucchiara BL, Liebeskind DS, Kasner SE. Utility of the NIH Stroke Scale as a Predictor of Hospital Disposition. Stroke 2003; 34(1):134-137.

8. Caneda MAG, Fernandes JG, Almeida AG, Mugnol FE. Confiabilidade de escalas de comprometimento neurológico em pacientes com acidente vascular. Arq. Neuropsiquiatr. 2006; 64(3a):690-697.

9. Kasner SE. Clinical interpretation and use of stroke scales. Lancet N eurol 2006; 5(7):603-612.

10. Riberto M, M iyazaki MH, Jucá SSH, Sakamoto H, Pinto PPN, Battistella LR. Validação da Versão Brasileira da M edida de Independência Funcional. Acta Fisiatr 2004; 11(2):72-76.

11. Silva MCR, Oliveira RJ, Conceição MIG. Efeitos da natação sobre a independência funcional de pacientes com lesão medular. Rev Bras M ed Esporte 2005; 11(4):251-256.

12. Kelly-Hayes M, Beiser A, Kase CS, Scaramucci A, D'Agostino RB, Wolf PA. The influence of gender and age on disability following ischemic stroke: the Framingham study. J Stroke Cerebrovasc Dis 2003; 12(3):119-126.

13. Sokrab TEO, Sid-Ahmed FM, Idris MNA. Acute stroke type, risk factors and early outcome in a developing country: A view from Sudan using a hospital-base sample. J Stroke Cerebrovasc Dis 2002; $11(2): 63-65$.

14. Kelly PJ, Furie KL, Shafqat S, Rallis N, Chang Y, Stein J. Functional recovery following rehabilitation after hemorrhagic and ischemic stroke. Arch Phys M ed Rehabil 2003; 84(7):968-972.

15. Schepers VP, Ketelaar M, Visser-M eily AJ, de Groot $V$, Twisk JW, Lindeman E. Functional recovery differs between ischaemic and haemorrhagic stroke patients. J Rehabil M ed. 2008; 40(6):487-489.

16. Paolucci S, Antonucci G, Grasso MG, Bragoni M, Coiro $P$, Angelis $D$, Fusco FR, Morelli $D$, Venturiero V, Troisi E, Pratesi L. Functional outcome of ischemic and hemorrhagic stroke patients after inpatient rehabilitation. Stroke 2003; 34(12):2861-2865. 
17. 17. Musicco M, Emberti L, Nappi G, Caltagirone C. Early and long-term outcome of rehabilitation in stroke patients: The role of patient characteristics, time of initiation, and duration of interventions. Arch Phys Med Rehabil. 2003; 84(4):551-558.

18. Patel MD, Coshall C, Rudd AG, Wolfe CD. Cognitive impairment after stroke: clinical determinants and its associations with long-term stroke outcomes. J Am Geriatr Soc 2002; 50(4):700-706

19. Conforto $A B$, Paulo RB, Patroclo CB, Pereira SLA, M iyahara HS, Fonseca CB, Yamamoto FI, Marchiori $P E$, Evaristo $E F$, Scaff $M$. Stroke management in a university hospital in the largest South American city. Arq Neuropsiquiatr. 2008; 66(2-B):308-311.

20. Pires SL, Gagliardi RJ, Gorzoni ML. Estudo das frequências dos principias fatores de risco para Acidente Vascular Cerebral em idosos. Arq Neuropsiquiatr. 2004; 62 (3B):844-851.

21. Voos MC, Ribeiro do Valle LE. Estudo comparativo entre a relação do hemisfério acometido no acidente vascular encefálico e a evolução funcional em indivíduos destros. Rev Bras Fisioter. 2008; $12(2): 113-120$

Artigo apresentado em 07/08/2008

Aprovado em 15/01/2009

Versão final apresentada em 26/01/2009 